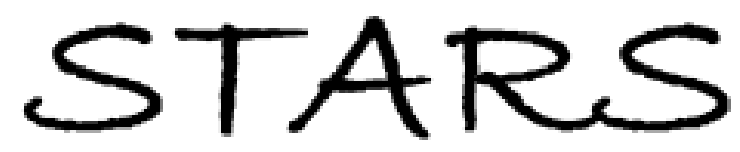

University of Central Florida

STARS

$1-1-2004$

\title{
Model for a partially coherent Gaussian beam in atmospheric turbulence with application in Lasercom
}

Olga Korotkova

Larry C. Andrews

University of Central Florida

Ronald L. Phillips

University of Central Florida

Find similar works at: https://stars.library.ucf.edu/facultybib2000

University of Central Florida Libraries http://library.ucf.edu

This Article; Proceedings Paper is brought to you for free and open access by the Faculty Bibliography at STARS. It has been accepted for inclusion in Faculty Bibliography 2000 s by an authorized administrator of STARS. For more information, please contact STARS@ucf.edu.

\section{Recommended Citation}

Korotkova, Olga; Andrews, Larry C.; and Phillips, Ronald L., "Model for a partially coherent Gaussian beam in atmospheric turbulence with application in Lasercom" (2004). Faculty Bibliography 2000s. 4502.

https://stars.library.ucf.edu/facultybib2000/4502

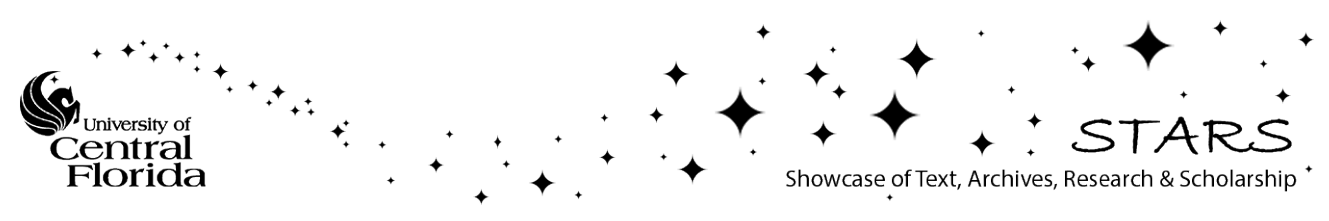




\section{Model for a partially coherent Gaussian beam in atmospheric turbulence with application in Lasercom}

\author{
Olga Korotkova, MEMBER SPIE \\ Larry C. Andrews, FELLOW SPIE \\ University of Central Florida \\ Department of Mathematics \\ Orlando, Florida 32816 \\ Ronald L. Phillips, FELLOW SPIE \\ University of Central Florida \\ Florida Space Institute \\ MS: FSI, Kennedy Space Center, \\ Florida 32899
}

\begin{abstract}
Analytic expressions for the mutual coherence function (MCF) and the scintillation index of a partially coherent lowest order Gaussian beam wave propagating through the atmosphere (based on Kolmogorov spectrum model) are developed for the pupil plane of a receiving system. Partial coherence of the beam is modeled as a thin (complex) phase screen with Gaussian spectrum (Rytov theory and $A B C D$ ray matrices are applied). The relation between the second- and fourth-order statistics for a beam with any degree of coherence in the atmosphere is introduced with the help of "effective" beam parameters, deduced from the free-space MCF. In particular, the scintillation (in weak and strong atmospheric conditions), based on these parameters, is studied as a function of the diffuser's strength and that of the atmosphere. The model is applied for the calculation of the SNR and bit error rates (OOK modulation) of the communication link with diffuser at the transmitter and slow detection system. The improvement of bit error rates is observed in weak and strong atmospheric turbulence. In the weak regime, the optimal diffuser can be found. (c) 2004 Society of Photo-Optical Instrumentation Engineers. [DOI: 10.1117/1.1636185]
\end{abstract}

Subject terms: scintillation index; diffuser; atmospheric turbulence; signal-tonoise ratio; bit error rates; Lasercom.

Paper 030229 received May 13, 2003; revised manuscript received Jul. 22, 2003; accepted for publication Aug. 12, 2003. This paper is a revision of a paper presented at the SPIE conference on Free-Space Laser Communication and Laser Imaging 11, July 2002, Seattle, Washington, and a paper presented at the SPIE conference on Atmospheric Propagation, January 2003, San Jose, California. The papers presented there appear (unrefereed) in SPIE Proceedings Vol. 4821 and Vol. 4976.

\section{Introduction}

The interest in the spatially partially coherent beam as a tool improving the performance of laser communication systems was indicated recently in a number of publications. $^{1-9}$ Although it was theoretically understood before that partially coherent beams are affected less by atmospheric turbulence compared with perfectly coherent beams, ${ }^{10}$ the efficient mathematical tools are not yet developed to carry this analysis over to the design of optical systems for communication links with required quality. In this paper, we establish the theoretical model for the calculation of the scintillation index of the partially coherent Gaussian beam in turbulence and demonstrate that it can provide such a tool for optical systems of different complexity and for a variety of atmospheric spectrum models. First, we present a short overview of the literature.

Studies of the propagation of a partially coherent beam wave through atmospheric turbulence have been conducted over the past three decades by numerous researchers. ${ }^{10-31} \mathrm{~A}$ partially coherent source (spatially) can be generated in a number of different ways, including the placement of a diffuser at the laser transmitter of a quasi-monochromatic source. Most theoretical studies concerning a spatially partially coherent beam wave rely on a Gaussian Schell model for describing the partial coherence of the source beam. ${ }^{25,26}$ This model utilizes a Gaussian correlation function to describe the surface roughness of the diffuser, often leading to simplifications. However, in practice a diffuser with other spectral distribution function might be used so that it is preferable to have a spectrum-independent model.

The free-space second-order statistical characteristics of a partially coherent beam are quite easy to develop and are discussed in detail in Mandel and Wolf. ${ }^{25}$ Fourth-order statistics follow directly from second-order statistics only for the special case of a Gaussian field model.

Banakh et al. ${ }^{10,30}$ and Banakh and Buldakov ${ }^{29}$ showed that intensity fluctuations of the Gaussian beam propagating through atmospheric turbulence can decrease as the source spatial coherence decreases. In these papers, asymptotic results were derived for the weak and saturation atmospheric regimes (excluding the important case of the focusing regime). In particular, in Ref. 29, only the limiting case of quasi-incoherent radiation was considered; in Ref. 30 , the comparison of rigorous solution for the scintillation index of incoherent source with experimental data was made. To our knowledge there are no analytic results for fourth-order statistics in the literature for the general case of the beam with arbitrary degree of spatial coherence. 
The effects of a temporally partially coherent source were studied by Fante ${ }^{18}$ and by Baykal and Plonus. ${ }^{21}$

The conventional method for developing the mutual coherence function (MCF) of a spatially partially coherent source is to use the extended Huygens-Fresnel principle. From knowledge of the MCF, one can then infer the spot radius of the beam and the spatial coherence radius. Extension of the extended Huygens-Fresnel principle to fourthorder statistics, such as the scintillation index, leads to mathematical complexities in the resulting integration that necessitate various types of approximations. The extended Huygens-Fresnel principle was used by Banakh et al. ${ }^{10}$ and by Baykal and Plonus ${ }^{21}$ whereas Leader $^{16}$ used the Rayleigh-Sommerfeld scattering theory. Most theoretical calculations, however, were based on a quadratic approximation for the structure function. A quadratic phase structure function implies that only wave tilts are induced on the wave by turbulence, which can lead to results that are inconsistent with experimental data. ${ }^{27,28}$

In this paper, we present an approach based on $A B C D$ ray matrix theory and model the diffuser as a thin random phase screen that induces a complex phase perturbation (i.e., both amplitude and phase) on the transmitted wave at the source. ${ }^{31,32}$ We note that it is a different modeling from the well-known phase screen approach, where only phase perturbations are taken into account. Although our method is flexible about the spectrum model for the diffuser (in fact, any physically meaningful spectrum could be applied), in this paper we use a Gaussian power spectrum to make a comparison of our new results with those based on the conventional Gaussian Schell model.

Primarily, we focus our attention on the scintillation index of the beam because this is the most important statistic for practical applications (lasercom, laser radar systems, etc.); however, other second- and fourth-order statistics of the wave field can be similarly calculated (refer to Chap. 8 of Ref. 32).

The other important generalization that our approach provides is the ability to utilize any of known atmospheric spectrum models ${ }^{32}$ including inner and/or outer scales. In this study (for the case of weak atmospheric turbulence), we restrict ourselves to the Kolmogorov spectrum model for illustration purposes. Analytic results for the scintillation index of a partially coherent beam valid in weak atmospheric fluctuations will be then extended to all atmospheric conditions with the help of a theory developed by Andrews et al. ${ }^{33,34}$

Therefore, none of the previously existing restrictions on the degree of spatial coherence of the beam, diffuser, and/or atmospheric spectrum models and the atmospheric regime are present in our model so that the continuous dependence of the scintillation index on the source coherence (strength of the diffuser) and strength of the atmospheric turbulence can be analyzed.

Note also that the $A B C D$ ray matrix can be calculated for the arbitrary optical system so that the scintillation index and other statistics can be derived at any distance from the transmitter after passing through any combination of optical elements, in particular, image plane analysis becomes possible. $^{35}$

Section 2 discusses the model and defines the Gaussian beam parameters for free-space propagation. Section 3 pre-

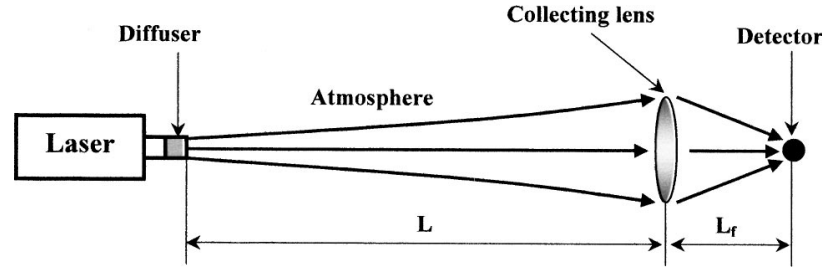

Fig. 1 Propagation of a partially coherent beam.

sents the derivation of the mutual coherence function of a partially coherent beam in free space. In Sec. 4 the effective beam parameters of partially coherent beam are defined and applied in the calculation of longitudinal and radial components of the scintillation index in weak and strong atmospheric turbulence (including the focusing regime). The application of the model for a communication link is demonstrated in Sec. 5, where the SNR and bit error rates are calculated.

\section{Basic Model and Beam Parameters}

A schematic diagram of the diffuser model and propagation channel for the partially coherent beam is shown in Fig. 1. We assume the transmitted beam wave in absence of the diffuser is a $\mathrm{TEM}_{00}$ Gaussian beam wave characterized by beam parameters

$\Theta_{0}=1-\frac{L}{F_{0}}, \quad \Lambda_{0}=\frac{2 L}{k W_{0}^{2}}$,

where $k=2 \pi / \lambda$ is the laser wave number, $\lambda$ (in meters) is wavelength, $L$ (in meters) is propagation distance to the collecting (Gaussian) lens, $F_{0}$ (in meters) is the phase front radius of curvature, and $W_{0}$ (in meters) is the laser exit aperture radius.

Following Andrews and Phillips, ${ }^{32}$ we introduce parameters $\Lambda_{1}$ and $\Theta_{1}$ for the beam incident on the collecting lens (in the pupil plane), which characterize the unperturbed incident beam spot size radius $W_{1}$ and phase front radius of curvature $F_{1}$ :

$\Lambda_{1}=\frac{2 L}{k W_{1}^{2}}=\frac{\Lambda_{0}}{\Theta_{0}^{2}+\Lambda_{0}^{2}}, \quad \Theta_{1}=1+\frac{L}{F_{1}}=\frac{\Theta_{0}}{\Theta_{0}^{2}+\Lambda_{0}^{2}}$.

We assume the Gaussian lens at the receiver has radius $W_{G}$ and phase front radius of curvature $F_{G}$. After propagating through the lens to the detector located in the image plane at distance $L_{f}$ behind the lens, the beam has radius $W_{2}$ and radius of curvature $F_{2}$, which are characterized by beam parameters

$$
\begin{aligned}
& \Lambda_{2}=\frac{2 L_{f}}{k W_{2}^{2}}=\frac{L}{L_{f}}\left[\frac{\lambda_{1}+\Omega_{G}}{\left(L / L_{f}-L / F_{G}+\bar{\Theta}_{1}\right)^{2}+\left(\Lambda_{1}+\Omega_{G}\right)^{2}}\right], \\
& \Theta_{2}=1+\frac{L_{f}}{F_{2}}=\frac{L}{L_{f}}\left[\frac{L / L_{f}-L / F_{G}+\bar{\Theta}_{1}}{\left(L / L_{f}-L / F_{G}+\bar{\Theta}_{1}\right)^{2}+\left(\Lambda_{1}+\Omega_{G}\right)^{2}}\right],
\end{aligned}
$$


where $\bar{\Theta}_{1}=1-\Theta_{1}$ and the nondimensional parameter $\Omega_{G}$ is defined by

$\Omega_{G}=\frac{2 L}{k W_{G}^{2}}$.

In Eq. (4) we identify the image plane of the system by imposing the generalized lens law condition ${ }^{32} L / L_{f}$ $-L / F_{G}+\bar{\Theta}_{1}=0$, or $\Theta_{2}=0$.

We model the diffuser in front of the laser transmitter by a thin random phase screen. ${ }^{31,32}$ However, we characterize the phase screen by a power spectrum function rather than by a correlation function as in the Gaussian Schell model. And, because the lateral correlation radius $l_{c}$ (in meters) is the only effective scale size associated with the diffuser, we can characterize the diffuser by a single-scale Gaussian spectrum model

$\Phi_{s}(\kappa)=\frac{\left\langle n_{1}^{2}\right\rangle l_{c}^{3}}{8 \pi \sqrt{\pi}} \exp \left(-\frac{1}{4} l_{c}^{2} \kappa^{2}\right)$,

where $\kappa$ (in inverse meters) is wave number, and $l_{c}$ (in meters) is directly related to the variance $\sigma_{g}^{2}$ used in Refs. 1 and 25 to describe the partial coherence properties of the source, namely,

$l_{c}^{2}=2 \sigma_{g}^{2}$

The parameter $\left\langle n_{1}^{2}\right\rangle$ is the fluctuation in the index of refraction induced by the diffuser.

In the following, we introduce $q_{c}=L / k l_{c}^{2}$ as a convenient nondimensional parameter analogous to the atmospheric parameter $q=L / k \rho_{\mathrm{pl}}^{2}$ used in propagation studies, where $\rho_{\mathrm{pl}}$ is the spatial coherence radius of a plane wave in turbulence.

\section{Mutual Coherence Function in Free Space}

Referring to Fig. 1, the $A B C D$ ray matrix for the propagation path between the diffuser and pupil plane of the optical system is simply

$$
\left(\begin{array}{ll}
A & B \\
C & D
\end{array}\right)=\left(\begin{array}{ll}
1 & L \\
0 & 1
\end{array}\right) \text {. }
$$

The optical beam in the absence of the diffuser can be obtained from the generalized Huygens-Fresnel integral, which yields ${ }^{34}$

$$
\begin{aligned}
U_{0}(\mathbf{r}, L)= & -\frac{i k}{2 \pi B} e^{i k L} \iint \mathrm{d}^{2} s U_{0}(\mathbf{s}, 0) \\
& \times \exp \left[\frac{i k}{2 B}\left(A s^{2}-2 \mathbf{s} \cdot \mathbf{r}+D r^{2}\right)\right] \\
= & \frac{1}{A+i \alpha B} e^{i k L} \exp \left[-\frac{1}{2} \beta(L) k r^{2}\right],
\end{aligned}
$$

where $r=|\mathbf{r}|, s=|\mathbf{s}|, A D-B C=1$, and
$\beta(L)=\frac{\alpha D-i C}{A+i \alpha B}$.

Let us assume the optical field at the transmitter is a unitamplitude Gaussian beam wave with spot size $W_{0}$ and phase front radius of curvature $F_{0}$ described by beam parameters in Eq. (1). Based on the simple $A B C D$ matrix given by Eq. (8), the optical field Eq. (9) in the pupil plane in the absence of atmospheric turbulence is

$$
\begin{aligned}
U(\mathbf{r}, L)= & U_{0}(\mathbf{r}, L) \exp \left[\Psi_{s}(\mathbf{r}, L)\right] \\
= & \left(\Theta_{1}+\Lambda_{1}\right) \exp \left[i k L+\frac{i k}{2 L}\left(\bar{\Theta}_{1}+i \Lambda_{1}\right) r^{2}\right] \\
& \times \exp \left[\Psi_{s}(\mathbf{r}, L)\right],
\end{aligned}
$$

where $\Psi_{s}$ is the complex phase perturbation caused by the diffuser. By using the thin phase screen model developed by Andrews and Phillips ${ }^{32}$ Andrews et al., ${ }^{33}$ we find that the MCF of the Gaussian beam in the pupil plane is given by*

$$
\begin{aligned}
\Gamma_{\text {diff }}\left(\mathbf{r}_{1}, \mathbf{r}_{2}, L\right)= & \left\langle U\left(\mathbf{r}_{1}, L\right) U^{*}\left(\mathbf{r}_{2}, L\right)\right\rangle \\
= & \Gamma_{0}\left(\mathbf{r}_{1}, \mathbf{r}_{2}, L\right) \exp \left\{-4 \pi^{2} k^{2} \Delta z \int_{0}^{\infty} \kappa \Phi_{s}(\kappa)\right. \\
& \times\left[1-\exp \left(\Lambda_{1} L \kappa^{2} / k\right)\right. \\
& \left.\left.\times J_{0}\left(\kappa\left|\Theta_{1} \mathbf{p}-2 i \Lambda_{1} \mathbf{r}\right|\right)\right] \mathrm{d} \kappa\right\}
\end{aligned}
$$

where $\Delta z$ is the thickness of the phase screen, $J_{0}(x)$ is a Bessel function, $r=(1 / 2)\left(\mathbf{r}_{1}+\mathbf{r}_{2}\right), \quad \mathbf{p}=\mathbf{r}_{1}-\mathbf{r}_{2}, \quad r=|\mathbf{r}|, \quad \rho$ $=|\mathbf{p}|$, and $\Gamma_{0}\left(\mathbf{r}_{1}, \mathbf{r}_{2}, L\right)$ is the MCF in the absence of the diffuser defined by

$\Gamma_{0}\left(\mathbf{r}_{1}, \mathbf{r}_{0}, L\right)=\frac{W_{0}^{2}}{W_{1}^{2}} \exp \left(-\frac{2 r^{2}}{W_{1}^{2}}-\frac{\rho^{2}}{2 W_{1}^{2}}-i \frac{k}{F_{1}} \mathbf{p} \cdot \mathbf{r}\right)$

Note that integration along the propagation path is not required in this thin phase screen model. By rearranging terms, Eq. (12) can also be written in the form ${ }^{32}$

$$
\begin{aligned}
\Gamma_{\text {diff }}\left(\mathbf{r}_{1}, \mathbf{r}_{2}, L\right)= & \Gamma_{0}\left(\mathbf{r}_{1}, \mathbf{r}_{2}, L\right) \exp \left[\sigma_{r, \text { diff }}^{2}\left(\mathbf{r}_{1}, L\right)\right. \\
& \left.+\sigma_{r, \text { diff }}^{2}\left(\mathbf{r}_{2}, L\right)\right] \exp \left[-T_{\text {diff }}(L)\right] \\
& \times \exp \left[-\frac{1}{2} \Delta_{\text {diff }}\left(\mathbf{r}_{1}, \mathbf{r}_{2}, L\right)\right],
\end{aligned}
$$

where each radial term $\sigma_{r \text {, diff }}^{2}(\mathbf{r}, L)$ is linked to a change in the mean intensity profile, $T_{\text {diff }}(L)$ describes the longitudinal or on-axis change in mean intensity, and

\footnotetext{
*Note that the function defined by Eq. (12) is usually called the MCF only by scientists working in areas of atmospheric propagation and lasercom systems. However, this is only the spatial component of the standard MCF defined in classic literature (the temporal counterpart is ignored due to the assumption of a quasi-monochromatic beam). Therefore, in our notation $\Gamma_{\text {diff }}\left(\mathbf{r}_{1}, \mathbf{r}_{2}, L\right)$ is the same as the mutual intensity $J\left(\mathbf{r}_{1}, \mathbf{r}_{2}, L\right)$ introduced in Mandel and Wolf ${ }^{25}$ or $J_{12}\left(\mathbf{r}_{1}, \mathbf{r}_{2}, L\right)$ by Goodman. ${ }^{31}$
} 
$\operatorname{Re}\left[\Delta_{\text {diff }}\left(\mathbf{r}_{1}, \mathbf{r}_{2}, L\right)\right]=D_{\text {diff }}\left(\mathbf{r}_{1}, \mathbf{r}_{2}, L\right)$ is the wave structure function (WSF). We use Re to denote the real part of the expression.

If we use the Gaussian spectrum [Eq. (6)], each radial component of the MCF [Eq. (14)] reduces to

$$
\begin{aligned}
\sigma_{r, \text { diff }}^{2}(\mathbf{r}, L)= & 2 \pi^{2} k^{2} \Delta z \int_{0}^{\infty} \kappa \Phi_{s}(\kappa) \exp \left(-\Lambda_{1} L \kappa^{2} / k\right) \\
& \times\left[I_{0}\left(2 \Lambda_{1} r \kappa\right)-1\right] \mathrm{d} \kappa \\
= & \frac{\sqrt{\pi}\left\langle n_{1}^{2}\right\rangle k^{2} l_{c} \Delta z}{2\left(1+4 \Lambda_{1} q_{c}\right)}\left[\exp \left(\frac{4 \Lambda_{1}^{2} r^{2}}{l_{c}^{2}\left(1+4 \Lambda_{1} q_{c}\right)}\right)-1\right],
\end{aligned}
$$

where $I_{0}(x)=J_{0}(i x)$. To equate our results with those based on a Gaussian Schell model, we introduce the normalization

$\frac{\sqrt{\pi}\left\langle n_{1}^{2}\right\rangle k^{2} l_{c} \Delta z}{1+4 \Lambda_{1} q_{c}}=1$

Then, combined with a small argument approximation for the exponential function consistent with the Rytov theory, we obtain the expression

$\sigma_{r, \mathrm{diff}}^{2}(\mathbf{r}, L)=\frac{2 \Lambda_{1}^{2} r^{2}}{l_{c}^{2}\left(1+4 \Lambda_{1} q_{c}\right)}=\frac{4 \Lambda_{1} q_{c}}{1+4 \Lambda_{1} q_{c}}\left(\frac{r^{2}}{W_{1}^{2}}\right)$

Similarly, the longitudinal component of the MCF is

$$
\begin{aligned}
T_{\text {diff }}(L) & =4 \pi^{2} k^{2} \Delta z \int_{0}^{\infty} \kappa \Phi_{s}(\kappa)\left[1-\exp \left(-\Lambda_{1} L \kappa^{2} / k\right)\right] \mathrm{d} \kappa \\
& =4 \Lambda_{1} q_{c}
\end{aligned}
$$

where we have again used the normalization of Eq. (16) and small argument approximation. The remaining quantity $\Delta_{\text {diff }}\left(\mathbf{r}_{1}, \mathbf{r}_{2}, L\right)$ in Eq. (14) has both real and imaginary parts given by

$$
\begin{aligned}
\Delta_{\text {diff }}\left(\mathbf{r}_{1}, \mathbf{r}_{2}, L\right)= & 4 \pi^{2} k^{2} \Delta z \int_{0}^{\infty} \kappa \Phi_{s}(\kappa) \exp \left(-\Lambda_{1} L \kappa^{2} / k\right) \\
& \times\left[I_{0}\left(2 \Lambda_{1} r_{1} \kappa\right)+I_{0}\left(2 \Lambda_{1} r_{2} \kappa\right)\right. \\
& \left.-2 J_{0}\left(\kappa\left|\Theta_{1} \mathbf{p}-2 i \Lambda_{1} \mathbf{r}\right|\right)\right] \mathrm{d} \kappa \\
= & 2\left(\frac{\Theta_{1}^{2}+\Lambda_{1}^{2}}{1+4 \Lambda_{1} q_{c}}\right) \frac{\rho^{2}}{l_{c}^{2}}-\frac{4 i \Theta_{1} \Lambda_{1} \mathbf{r} \cdot \mathbf{p}}{\left(1+4 \Lambda_{1} q_{c}\right) l_{c}^{2}}
\end{aligned}
$$

the last step of which is a result of using the normalization of Eq. (16) and small argument approximation. The combination of results taken from Eqs. (12) to (19) yields the following form for the MCF

$$
\begin{aligned}
\Gamma_{\text {diff }}(\mathbf{r}, \mathbf{p}, L)= & \left(\frac{\Theta_{1}^{2}+\Lambda_{1}^{2}}{1+4 \Lambda_{1} q_{c}}\right) \exp \left[-\frac{\left(2 r^{2}+\rho^{2}\right) / 2}{W_{1}^{2}\left(1+4 \Lambda_{1} q_{c}\right)}\right] \\
& \times \exp \left[-\left(\frac{\Theta_{1}^{2}+\Lambda_{1}^{2}}{1+4 \Lambda_{1} q_{c}}\right) \frac{\rho^{2}}{l_{c}^{2}}\right] \exp \\
& \times\left[\frac{i k}{L}\left(\frac{1-\Theta_{1}+4 \Lambda_{1} q_{c}}{1+4 \Lambda_{1} q_{c}}\right) \mathbf{r} \cdot \mathbf{p}\right] .
\end{aligned}
$$

Although we use different notation, it is easy to show that this expression for the MCF is in exact agreement with that based on a Gaussian Schell model and a quasimonochromatic Gaussian beam wave (e.g., see p. 280 in Mandel and $\mathrm{Wolf}^{25}$ ). All second-order statistics based on the MCF such as beam intensity, coherence radius, beam spread, etc. can also be derived ${ }^{35}$ from Eq. (20).

The calculation of the MCF in the plane of the photodetector (image plane) can be made similarly. ${ }^{35}$ In this case the $A B C D$ ray matrix takes the form

$$
\begin{aligned}
\left(\begin{array}{ll}
A & B \\
C & D
\end{array}\right) & =\left(\begin{array}{cc}
1 & L_{f} \\
0 & 1
\end{array}\right)\left(\begin{array}{cc}
1 & 0 \\
i \alpha_{G} & 1
\end{array}\right)\left(\begin{array}{cc}
1 & L \\
0 & 1
\end{array}\right) \\
& =\left(\begin{array}{cc}
1+i \alpha_{G} L_{f} & L+L_{f}\left(1+i \alpha_{G} L\right) \\
i \alpha_{G} & 1+i \alpha_{G} L
\end{array}\right),
\end{aligned}
$$

where $\alpha_{G}=2 /\left(k W_{G}^{2}\right)+i\left(1 / F_{G}\right)$.

\section{Scintillation Index}

Next we calculate the scintillation index caused by the combination of diffuser and atmospheric turbulence. In calculating the scintillation index, it is the integrated intensity that we must consider, taking into account the response time $\tau_{d}$ of the detector and the coherence time $\tau_{s}$ of the source. That is, the coherence time of a quasimonochromatic laser source is $\tau_{s} \cong 1 / B$, where $B$ is the bandwidth of the source. If the source coherence time is much smaller than the detector's integration time interval $\tau_{d}$, i.e., $\tau_{s} \ll \tau_{d}$ (slow detector), temporal averaging of the fluctuating intensity occurs, which reduces the scintillation level through source aperture averaging. ${ }^{21,24}$ In the case of a fast detector $\tau_{s} \gg \tau_{d}$, the detector is sensitive to intensity fluctuations of the source as well as those caused by the atmospheric turbulence. In both cases above the coherence time $\tau_{a}$ of the atmospheric turbulence is slow with respect to both $\tau_{s}$ and $\tau_{d}$, i.e.,

$\tau_{s} \ll \tau_{d} \ll \tau_{\alpha}$,

for a slow detector, and

$\tau_{d} \ll \tau_{s} \ll \tau_{a}$,

for a fast detector. In this paper, we restrict ourselves to the case of slow detector only.

\subsection{Longitudinal Component}

In this section we develop the on-axis or longitudinal atmospheric scintillation index. The longitudinal component of 
the scintillation index at the collecting lens will be used in Sec. 5 for the calculation of the flux variance of intensity fluctuations at the photodetector.

In the presence of atmospheric turbulence, we must take into account some scattering properties caused by the diffuser. Namely, each scattering center (speckle cell size) associated with the spatial correlation radius $l_{c}$ of the diffuser surface acts like a separate beam coherence center within the original beam source diameter. Hence, the diffuser creates an "array of independent scattering centers," the number of which is often approximated by ${ }^{16}$

$N_{s}=1+\frac{2 W_{0}^{2}}{l_{c}^{2}}=1+\frac{4 q_{c}}{\Lambda_{0}}$.

Thus, the number of speckle cells is of the order of unity for a weak diffuser and increases rapidly as the correlation radius $l_{c}$ decreases. Taking the scattering properties of the diffuser into account, we find it useful to model the beam at the receiver by an "effective diffuser beam" (denoted later by the subscript $e$ ) that changes in accordance with the roughness or strength of the diffuser.

We can characterize the effective diffuser beam at the receiver by replacing the standard beam parameters $\Theta_{1}$ and $\Lambda_{1}$ that arise in the absence of the diffuser with a set of effective beam parameters $\Theta_{e}$ and $\Lambda_{e}$. To identify these effective beam parameters, we simply compare parameters of the MCF in the absence of the diffuser with parameters associated with the MCF in the presence of the diffuser. For example, the MCF in the absence of the diffuser, scaled by the on-axis intensity, is given by

$\frac{\Gamma_{0}(\mathbf{r}, \mathbf{p}, L)}{\Gamma_{0}(0,0, L)}=\exp \left(-\frac{\Lambda_{1} k r^{2}}{L}-\frac{\Lambda_{1} k \rho^{2}}{4 L}+\frac{i k \bar{\Theta}_{1}}{L} \mathbf{r} \cdot \mathbf{p}\right)$,

which identifies beam parameters $\bar{\Theta}_{1}=-L / F_{1}$ and $\Lambda_{1}$ $=2 L / k W_{1}^{2}$. The scaled MCF in the presence of the diffuser can be rearranged in the form [see Eq. (20)]

$$
\begin{aligned}
\frac{\Gamma_{\text {diff }}(\mathbf{r}, \mathbf{p}, L)}{\Gamma_{\text {diff }}(0,0, L)}= & \exp \left[-\frac{\Lambda_{1} k r^{2}}{\left(1+4 \Lambda_{1} q_{c}\right) L}-\frac{\Lambda_{1} N_{s} k \rho^{2}}{4\left(1+4 \Lambda_{1} q_{c}\right) L}\right. \\
& \left.+\frac{i k\left(\bar{\Theta}+4 \Lambda_{1} q_{c}\right)}{\left(1+4 \Lambda_{1} q_{c}\right) L} \mathbf{r} \cdot \mathbf{p}\right] .
\end{aligned}
$$

On comparing similar terms appearing in Eqs. (25) and (26), the effective radius of curvature of the beam can be identified in Eq. (26) by the last term involving the complex exponential function, namely

$\bar{\Theta}_{e}=-\frac{L}{F_{e}}=\frac{\bar{\Theta}_{1}+4 \Lambda_{1} q_{c}}{1+4 \Lambda_{1} q_{c}}$.

This quantity is a function of the strength of the diffuser $q_{c}$, which in the limiting case of a strong diffuser $q_{c} \rightarrow \infty$ $\left(l_{c} \rightarrow 0\right)$, yields $\bar{\Theta}_{e}=1$ (i.e., the phase front radius of curvature $F_{e}$ approaches $-L$, similar to the case of a focused beam). For a weak diffuser $q_{c} \rightarrow 0\left(l_{c} \rightarrow \infty\right)$, we are led to $\bar{\Theta}_{e}=\bar{\Theta}_{1}$ (i.e., $F_{e}$ reduces to its free-space value $F_{1}$ in the absence of the diffuser). Hence, in the development here, we will formally replace the parameter $\Theta_{1}$ with the effective beam parameter

$\Theta_{e}=1+\frac{L}{F_{e}}=\frac{\Theta_{1}}{1+4 \Lambda_{1} q_{c}}=\frac{\Theta_{0}}{\Theta_{0}^{2}+\Lambda_{0}^{2} N_{s}}$,

where we have related $\Theta_{e}$ to receiver plane beam parameters and to transmitter plane beam parameters. The quantity $N_{s}$ is the number of speckle cells [Eq. (24)].

For the effective beam parameter $\Lambda_{e}$ we are faced with a different situation. Namely, the term involving $\rho^{2}$ can be associated with longitudinal characteristics of the beam wave induced by the diffuser (rather than specific points in the beam). In this case, we define

$\Lambda_{e}=\frac{\Lambda_{1} N_{s}}{1+4 \Lambda_{1} q_{c}}=\frac{\Lambda_{0} N_{s}}{\Theta_{0}^{2}+\Lambda_{0}^{2} N_{s}}$,

limiting cases of which lead to

$\Lambda_{e} \sim\left\{\begin{array}{cc}\Lambda_{1}, & q_{c} \rightarrow 0 \\ 1 / \Lambda_{0}, & q_{c} \rightarrow \infty\end{array}\right.$.

That is, for a weak diffuser the effective beam parameter of Eq. (29) reduces to parameter $\Lambda_{1}$, whereas for a strong diffuser it reduces to $1 / \Lambda_{0}$, the latter of which can be associated with transmitter aperture averaging. ${ }^{36}$ However, on the basis of the radial behavior of the beam or effective spot size, we can identify an effective parameter $\Lambda_{e}$ with the term involving $r^{2}$ in Eq. (26). Doing so, we would obtain the same expression as Eq. (29), but with $N_{s}=1$ in the numerator terms. Because we are concerned here with the longitudinal component of the scintillation index, we use Eq. (29) to define $\Lambda_{e}$.

Based on the preceding observations, we claim the longitudinal component of the scintillation index in the presence of a diffuser is defined under the Rytov approximation by $^{32}$

$$
\begin{aligned}
\sigma_{1, \text { weak }}^{2}(0, L)= & 8 \pi^{2} k^{2} L \int_{0}^{1} \int_{0}^{\infty} \kappa \Phi_{n}(\kappa) \exp \left(-\frac{\Lambda_{e} L \kappa^{2} \xi}{k}\right) \\
& \times\left\{1-\cos \left[\frac{L \kappa^{2}}{k} \xi\left(1-\bar{\Theta}_{e} \xi\right)\right]\right\} \mathrm{d} \kappa \mathrm{d} \xi,
\end{aligned}
$$

where $\bar{\Theta}_{e}=1-\Theta_{e}$. Based on a Kolmogorov spectrum, this integral yields ${ }^{32}$

$$
\begin{aligned}
\sigma_{1, \text { weak }}^{2}(0, L) \equiv & \sigma_{B}^{2} \\
= & 3.86 \sigma_{1}^{2} \operatorname{Re}\left[\mathrm{i}^{5 / 6}{ }_{2} F_{1}\left(-\frac{5}{6}, \frac{11}{6} ; \frac{17}{6} ; \bar{\Theta}_{e}+i \Lambda_{e}\right)\right. \\
& \left.-\frac{11}{16} \Lambda_{e}^{5 / 6}\right]
\end{aligned}
$$

where ${ }_{2} F_{1}(a, b ; c ; x)$ is a hypergeometric function. ${ }^{34}$ 


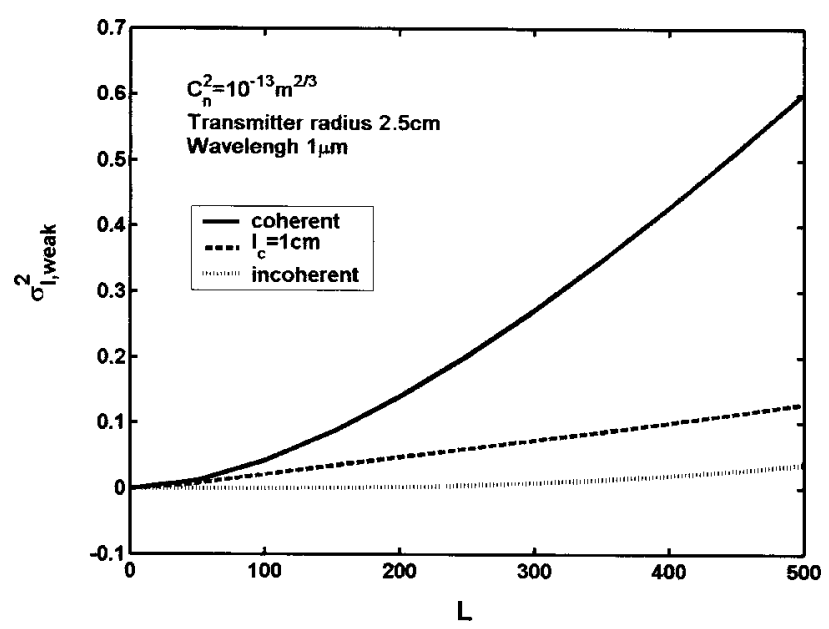

Fig. 2 Effective scintillation index of a partially coherent beam in weak atmosphere versus strength of turbulence (Rytov variance $\sigma_{1}$ ) and nondimensional diffuser's correlation $q_{c}$.

Numerical values deduced from Eq. (32) are essentially the same as those obtained from Eq. (6) in Banakh et al. ${ }^{10}$ In Fig. 2 we compare the scintillation index [Eq. (32)] as a function of propagation distance $L$ for a Gaussian beam wave propagating through atmospheric turbulence in the absence of the diffuser $l_{c}=\infty$ (solid curve), for a moderate diffuser with $l_{c}=1 \mathrm{~cm}$ (dashed curve) and for a very strong diffuser $l_{c}=0$ (dashed curve). Input parameters are $W_{0}$ $=2.5 \mathrm{~cm}, \lambda=1 \mu \mathrm{m}$, and $C_{n}^{2}=10^{-13} \mathrm{~m}^{-2 / 3}$. For smaller values of $l_{c}$ the scintillation can be considerably reduced. However, the effect generally saturates for $l_{c}$ of the order of $1 \mathrm{~mm}$.

Equation (32) is restricted to weak fluctuations. Under strong fluctuation conditions, we can use the strong fluctuation theory developed by Andrews et al. ${ }^{32,34}$ which yields

$$
\begin{aligned}
\sigma_{1, \text { atm }}^{2}(0, L)= & \exp \left[\frac{0.49 \sigma_{B}^{2}}{\left(1+0.56 \sigma_{B}^{12 / 5}\right)^{7 / 6}}+\frac{0.51 \sigma_{B}^{2}}{\left(1+0.69 \sigma_{B}^{12 / 5}\right)^{5 / 6}}\right] \\
& -1
\end{aligned}
$$

where $\sigma_{B}^{2}$ is defined by Eq. (32).

In Fig. 3 we show Eq. (33) as a function of the Rytov variance $\sigma_{1}^{2}=1.23 C_{n}^{2} k^{7 / 6} L^{11 / 6}$ for fixed propagation distance $L=1 \mathrm{~km}$. There is a smooth transition of the scintillation index from one limiting case [coherent beam (no diffuser, $q_{c}=0$ ) to the other incoherent (strong diffuser, $\left.\left.q_{c}=\infty\right)\right]$. As the effect of the diffuser increases, the maximum value of the scintillation index does not change but occurs at larger values of the Rytov variance; therefore, the advantage of using partially coherent beams can be expected in weak or focusing atmospheric regimes.

Figure 4 displays the result [Eq. (33)] from a different perspective: the refractive index structure parameter is fixed $C_{n}^{2}=10^{-13} \mathrm{~m}^{2 / 3}$, but the propagation distance changes. We show three cases: coherent beam with $l_{c}=\infty$ (solid curve), partially coherent beam with $l_{c}=1 \mathrm{~cm}$ (dashed curve), and incoherent beam with $l_{c}=0$ (dotted

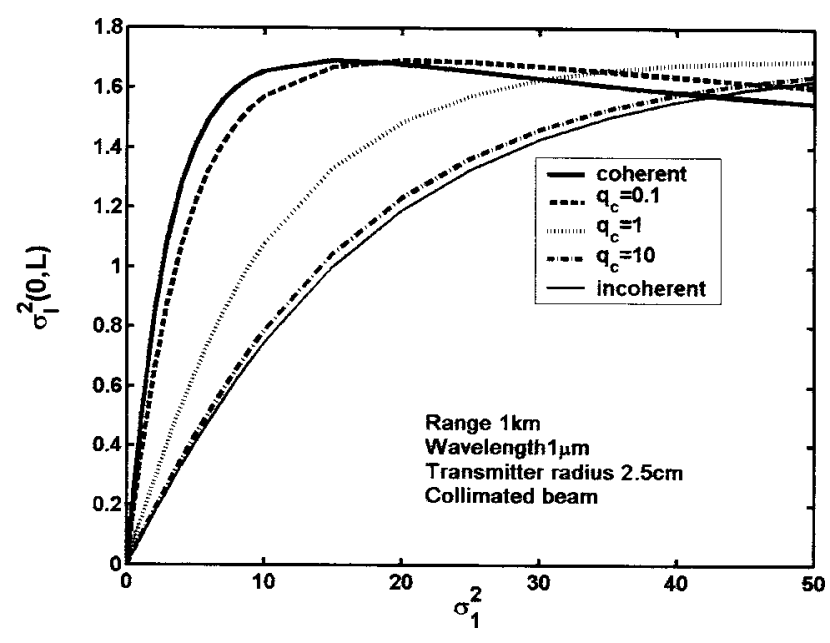

Fig. 3 Effective scintillation index of a partially coherent beam in the atmosphere versus strength of turbulence (Rytov variance $\sigma_{1}$ ) and nondimensional diffuser's correlation $q_{c}$.

curve). For short ranges (up to 3 to $4 \mathrm{~km}$ ) partially coherent and incoherent beams assume lower levels of the scintillation index compared with a coherent beam.

\subsection{Radial Component}

At a point in the beam wave off the optical axis, the scintillation index can be expressed as the sum of two components

$\sigma_{1}^{2}(\mathbf{r}, L)=\sigma_{1, r}^{2}(\mathbf{r}, L)+\sigma_{1}^{2}(0, L)$,

where the first component is the radial component and the second is the longitudinal component defined by Eq. (33). The radial component vanishes on the optical axis $(r=0)$ and, under weak fluctuation conditions in the absence of a diffuser, it is known that the radial component of the scintillation index is given by ${ }^{32}$

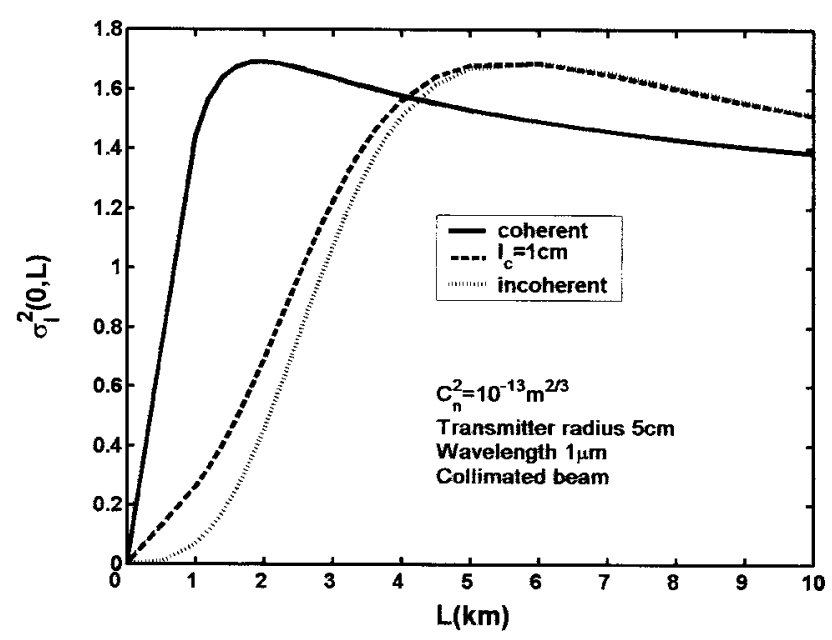

Fig. 4 Effective scintillation index of a partially coherent beam in the atmosphere versus the propagation distance $L$ and correlation distance $I_{c}$. 


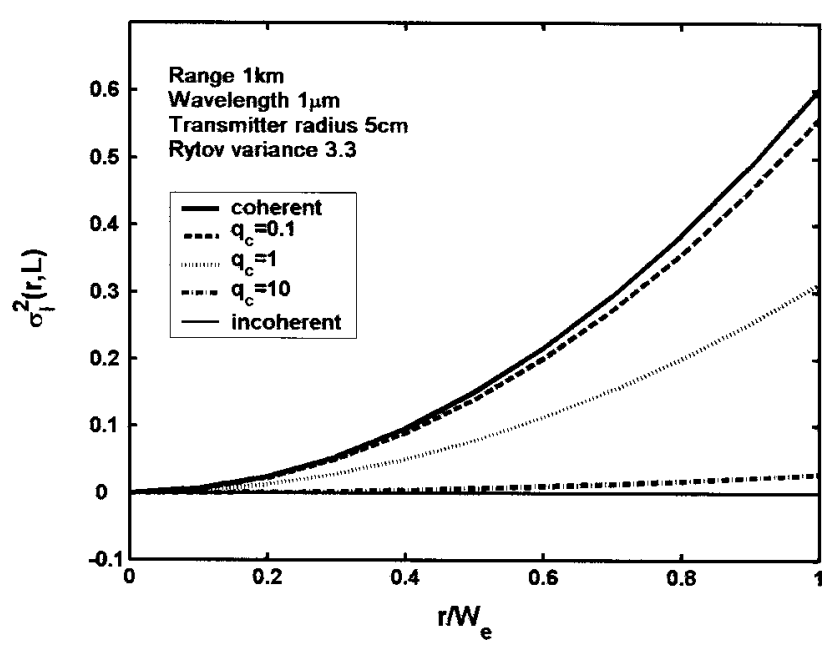

Fig. 5 Effective radial component of the scintillation index for different normalized correlations of the diffuser.

$\sigma_{1, r}^{2}(\mathbf{r}, L)=4.42 \sigma_{1}^{2} \Lambda_{1}^{5 / 6} \frac{r^{2}}{W_{1}^{2}}, \quad r<W_{1}, \quad \sigma_{1}^{2} \ll 1$.

It was shown in Ref. 35 that an additional diffraction caused by the atmosphere and diffuser together lead to an effective spot radius given by

$W_{e}=W_{1}\left(1+4 q_{c} \Lambda_{1}+1.63 \sigma_{1}^{12 / 5} \Lambda_{1}\right)^{1 / 2}$.

By following the approach in Refs. 34 and 37, we therefore make the beam parameter replacement

$\Lambda_{1} \Rightarrow \frac{\Lambda_{1}}{1+4 \Lambda_{1} q_{c}+1.63 \sigma_{1}^{12 / 5} \Lambda_{1}}$,

and formally deduce that

$$
\begin{aligned}
\sigma_{1, r}^{2}(\mathbf{r}, L)= & 4.42 \sigma_{1}^{2}\left(\frac{\Lambda_{1}}{1+4 \Lambda_{1} q_{c}+1.63 \sigma_{1}^{12 / 5} \Lambda_{1}}\right)^{5 / 6} \\
& \times \frac{r^{2}}{W_{1}^{2}\left(1+4 \Lambda_{1} q_{c}+1.63 \sigma_{1}^{12 / 5} \Lambda_{1}\right)}, \quad r<W_{1} .
\end{aligned}
$$

In both Eqs. (35) and (38), we have retained the restriction $r<W_{1}$, although this may be more restrictive than necessary. The full range of validity for these expressions has not yet been established. As the Rytov variance $\sigma_{1}^{2} \rightarrow \infty$, the radial component [Eq. (35)] eventually vanishes and the beam wave acts more and more like a propagating spherical wave.

Note that, for a fixed strength of atmospheric turbulence (i.e., $\sigma_{1}^{2}=$ const.), the radial component vanishes in the limit $l_{c} \rightarrow 0 \quad\left(q_{c} \rightarrow \infty\right)$. Similarly, for a fixed diffuser strength (i.e., $l_{c}=$ const.), the radial component vanishes as $\sigma_{1}^{2} \rightarrow \infty$. In Fig. 5 we plot the radial component of the scintillation index [Eq. (38)] versus $r / W_{e}$ for different values of $q_{c}$. The radial component of the scintillation index for the incoherent source is zero independently of the displacement $r$.

The radial component should generally be included into analysis when perfect alignment of the transmitter and the receiver systems is not possible.

\section{Application for Laser Communications}

The reduction of the on-axis scintillation index due to partial coherence of the source discussed in Sec. 4 alone would not be sufficient for the high-quality data transfer calculated in terms of the bit error rate (BER) in communication systems. However, the combination of partial coherence with large enough collecting lens can provide the required BER level. Following Ref. 34, the flux variance of the irradiance fluctuations (valid for all atmospheric conditions) at the detector plane calculated for the collecting lens with normalized radius $\Omega_{G}$ is

$$
\begin{aligned}
\sigma_{\text {irrad }}^{2}\left(L+L_{f}, \Omega_{G}\right)= & \exp \left\lfloor\sigma_{\ln x}^{2}\left(L+L_{f}, \Omega_{G}\right)\right. \\
& \left.+\sigma_{\ln y}^{2}\left(L+L_{f}, \Omega_{G}\right)\right\rfloor-1,
\end{aligned}
$$

where $\sigma_{\ln x}^{2}$ is the flux variance associated with large-scale fluctuations given by ${ }^{38}$

$$
\begin{aligned}
\sigma_{\ln x}^{2}\left(L+L_{f}, \Omega_{G}\right)= & 0.49 \sigma_{1}^{2}\left(\frac{\Omega_{G}-\Lambda_{e}}{\Omega_{G}+\Lambda_{e}}\right)^{2} \\
& \times R\left[\frac{\eta_{x}}{1+0.4 \eta_{x}\left(1+\Theta_{e}\right) /\left(\Lambda_{e}+\Omega_{G}\right)}\right]^{7 / 6},
\end{aligned}
$$

where $R=1 / 3-(1 / 2)\left(1-\Theta_{e}\right)+(1 / 5)\left(1-\Theta_{e}\right)^{2}$. The quantity $\eta_{x}$ in Eq. (40) is the normalized large-scale cutoff frequency determined by the asymptotic behavior of $\sigma_{\ln x}^{2}$ in weak turbulence and saturation regime ${ }^{36,38}$ :

$\eta_{x}=\frac{R^{-6 / 7}\left(\sigma_{B} / \sigma_{1}\right)^{12 / 7}}{1+0.56 \sigma_{B}^{12 / 5}}$.

The small-scale flux variance $\sigma_{\ln y}^{2}$ in Eq. (39) is similarly defined by

$\sigma_{\ln y}^{2}\left(L+L_{f}, \Omega_{G}\right)=\frac{1.27 \sigma_{1}^{2} \eta_{y}^{-5 / 6}}{1+0.4 \eta_{y} /\left(\Lambda_{1}+\Omega_{G}\right)}$,

where the corresponding cutoff frequency is

$\eta_{y}=3\left(\sigma_{1} / \sigma_{B}\right)^{12 / 5}\left(1+0.69 \sigma_{B}^{12 / 5}\right)$.

Figure 6 shows the flux variance [Eq. (39)] versus the normalized parameter of the collecting lens $\Omega_{G}$ [defined in Eq. (5)] for a perfectly coherent beam, several partially coherent beams with $q_{c}=0.1,1$, and 10 , and an incoherent beam. The propagation distance $L=1 \mathrm{~km}, \quad C_{n}^{2}$ $=10^{-13} \mathrm{~m}^{-2 / 3}$, leading to the Rytov variance $\sigma_{1}^{2}$ $=1.23 C_{n}^{2} L^{11 / 6} k^{7 / 6}=0.33$. We see that in the weak fluctuation regime there is a significant reduction of the flux, es- 


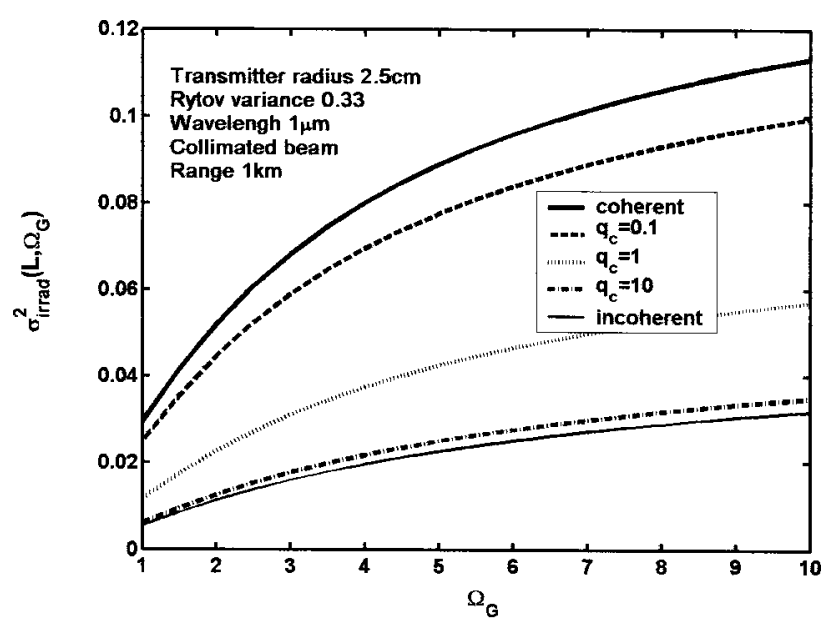

Fig. 6 Flux variance $\sigma_{\text {irrad }}^{2}\left(L+L_{f}, \Omega_{G}\right)$ as a function of the normalized radius of the collecting lens $\Omega_{G}$ for coherent and partially coherent beams in weak turbulence.

pecially for a point aperture $\left(\Omega_{G} \gg 1\right)$. With the increase of the collecting aperture size this effect decreases. In the analysis of the SNR and the BERs here we choose the collecting aperture radius $W_{G}=1 \mathrm{~cm}$ to concentrate primarily on the averaging effect due to the transmitter. Similar curves are generated in moderate turbulence $\left(\sigma_{1}^{2}=1.05\right)$ in Fig. 7 and in strong turbulence $\left(\sigma_{1}^{2}=7.0\right)$ in Fig. 8, but as the strength of turbulence grows the absolute reduction of flux variance deminishes.

\subsection{Signal-to-Noise Ratio (SNR)}

In free space, the signal to noise ratio $\mathrm{SNRC}_{0}$ of a coherent beam is conventionally defined by ${ }^{34}$

$\mathrm{SNRC}_{0}=\frac{i_{S}}{\sigma_{N}}$,

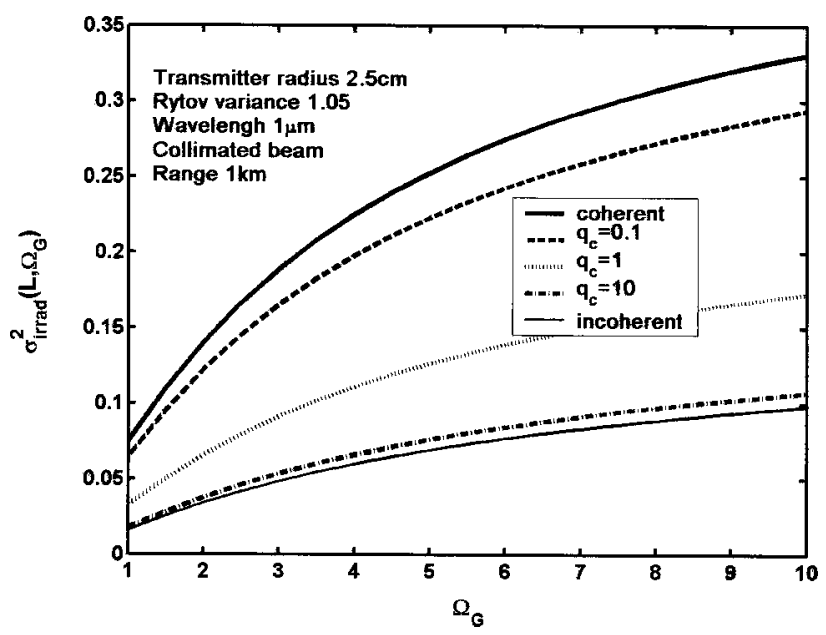

Fig. 7 Flux variance $\sigma_{\text {irrad }}^{2}\left(L+L_{f}, \Omega_{G}\right)$ as a function of the normalized radius of the collecting lens $\Omega_{G}$ for coherent and partially coherent beams in focusing regime.

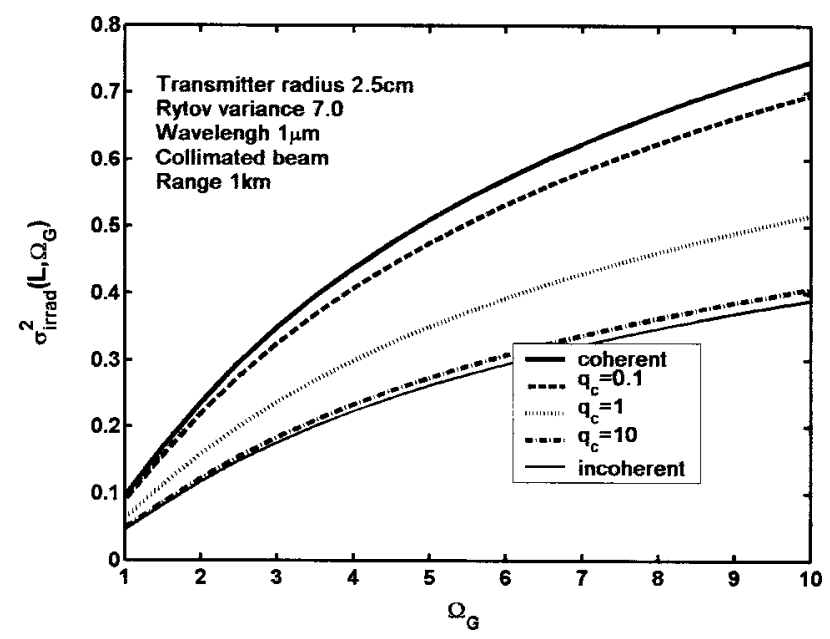

Fig. 8 Flux variance $\sigma_{\text {irrad }}^{2}\left(L+L_{f}, \Omega_{G}\right)$ as a function of the normalized radius of the collecting lens $\Omega_{G}$ for coherent and partially coherent beams in strong turbulence.

where $i_{S}$ is the received signal current (proportional to the transmitted power) and $\sigma_{N}$ is the standard deviation of the detector noise. Since partially coherent beams have greater divergence than coherent beams, the received power depends on the degree of coherence of the wave as well, i.e., more power is required for less coherent beams to sustain the same SNR as the perfectly coherent beam (of the same size and phase front radius of curvature) produces. Therefore in free space the relation between the $\mathrm{SNRC}_{0}$ and the SNR of a partially coherent beam, say, $\mathrm{SNRP}_{0}$ can be deduced from this power loss or, equivalently, from the beam size of partially coherent beam at the receiver ${ }^{38}$

$$
\begin{aligned}
\mathrm{SNRP}_{0}=\frac{\mathrm{SNRC}_{0}}{\left(P P_{0} / P C_{0}\right)^{1 / 2}} & =\frac{\mathrm{SNRC}_{0}}{\left(1+4 q_{c} \Lambda_{1}\right)^{1 / 2}} \\
& =\frac{i_{S}}{\sigma_{N}\left(1+4 q_{c} \Lambda_{1}\right)^{1 / 2}},
\end{aligned}
$$

where $P P_{0}$ is the received power of the partially coherent beam and $P C_{0}$ is the power of the coherent beam. The quantity $1+4 q_{c} \Lambda_{1}$ represents the relative beam spread due to the diffuser with strength ${ }^{38} q_{c}$.

In the atmosphere the standard definition of the mean SNR of a partially coherent beam can be adapted similarly $^{34,39}$

$$
\begin{aligned}
\langle\mathrm{SNRP}\rangle & =\frac{\mathrm{SNRC}_{0}}{\left(\langle P P\rangle / P P_{0}+\sigma_{\text {irrad }}^{2} \mathrm{SNRC}_{0}^{2}\right)^{1 / 2}} \\
& =\frac{\mathrm{SNRC}_{0}}{\left(1+4 q_{c} \Lambda_{1}+1.63 \sigma_{1}^{12 / 5} \Lambda_{1}+\sigma_{\text {irrad }}^{2} \mathrm{SNRC}_{0}^{2}\right)^{1 / 2}},
\end{aligned}
$$

where brackets are used for ensemble averaging, $\sigma_{1}^{2}$ is the Rytov variance, and flux variance $\sigma_{\text {irrad }}^{2}$ was defined by Eq. (39). 


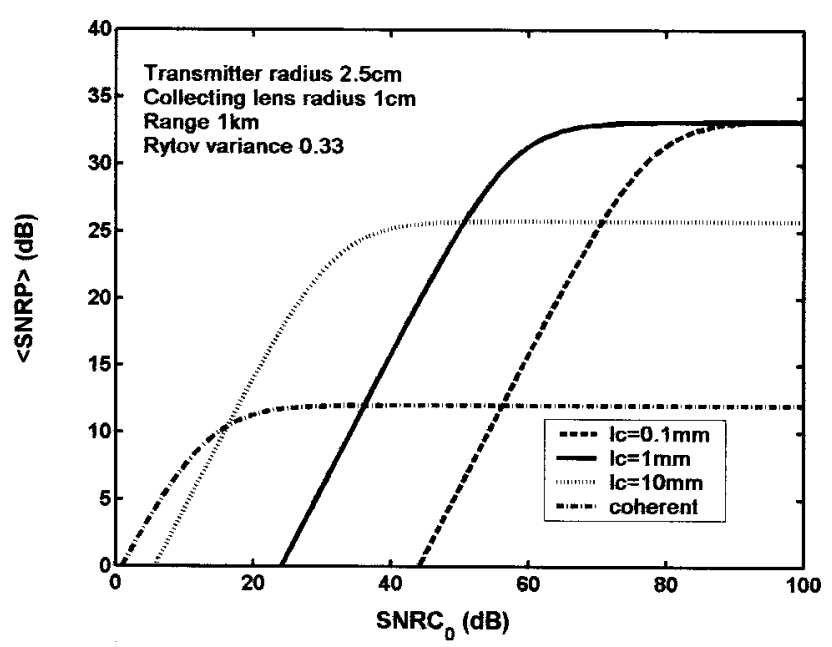

Fig. $9\langle\mathrm{SNRP}\rangle$ (in decibels) as a function of $\mathrm{SNRC}_{0}$ (in decibels) in weak turbulence for several values of $I_{c}$.

At first, note that Eq. (46) implies that regardless of the amount of the input power $\left(\mathrm{SNRC}_{0}\right)\langle\mathrm{SNRP}\rangle$ stays limited by the scintillation ${ }^{39}$ [in striking contrast with free space analysis of Eq. (45)], i.e.,

$$
\lim _{\mathrm{SNRC}_{0} \rightarrow \infty}\langle\mathrm{SNRP}\rangle=\frac{1}{\sigma_{\text {irrad }}} .
$$

The other important consequence of Eq. (46) stems from the fact that for given $\mathrm{SNRC}_{0}$ and atmospheric conditions the value of $\langle\mathrm{SNRP}\rangle$ is determined by two factors: power reduction $\langle P P\rangle / P P_{0}$ caused by beam divergence and flux variance $\sigma_{\text {irrad }}^{2}$. While the former term obviously deteriorates $\langle\mathrm{SNRP}\rangle$ the latter can improve it. That is, if $\sigma_{\text {irrad }}^{2}$ is reduced because of the diffuser, then $\langle\mathrm{SNRP}\rangle$ will increase independently of beam spreading, provided $\mathrm{SNRC}_{0}$ is sufficiently large according to Eq. (47).

Figures 9 to 11 show $\langle\mathrm{SNRP}\rangle$ (in decibels) is displayed as a function of $\mathrm{SNRC}_{0}$ (in decibels) in different atmo-

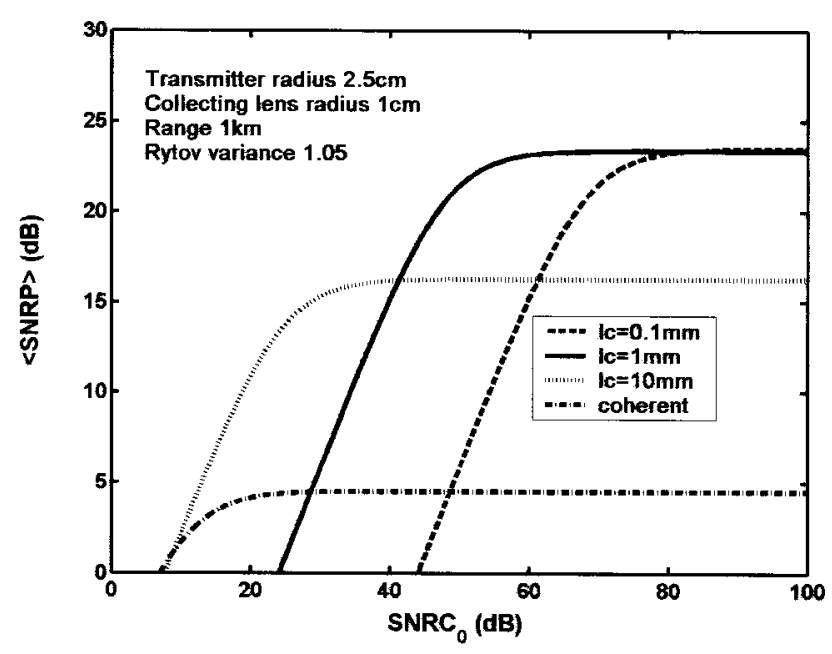

Fig. $10\langle\mathrm{SNRP}\rangle$ (in decibels) as a function of $\mathrm{SNRC}_{0}$ (in decibels) in the focusing regime for several values of $I_{c}$.

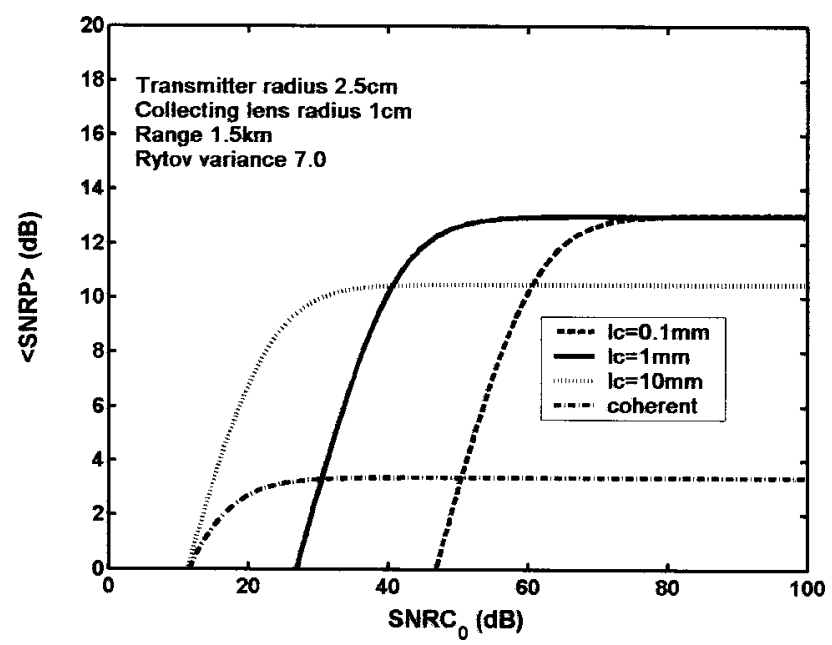

Fig. $11\langle\mathrm{SNRP}\rangle$ (in decibels) as a function of $\mathrm{SNRC}_{0}$ (in decibels) in strong turbulence for several values of $I_{c}$.

spheric conditions. We note that in all regimes the atmospheric SNR of a perfectly coherent beam (dashed-anddotted curves everywhere) is always below 21 to $22 \mathrm{~dB}$, which corresponds to conventionally accepted BER of the operating system (of the order of $10^{-9}$ ).

In the weak fluctuation regime (Fig. 9) the use of partial coherence provides a noticeable improvement of $\langle\mathrm{SNRP}\rangle$, namely, with $\mathrm{SNRC}_{0}$ in the range 40 to $50 \mathrm{~dB}$, it is possible to obtain $\langle\mathrm{SNRP}\rangle$ (in decibels) exceeding 21 to $22 \mathrm{~dB}$. (This can be explained with help of Fig. 6, where it is shown that the flux-variance of partially coherent beam in this regime takes sufficiently low values.) Also, from Fig. 9, the saturation effect [Eq. (47)] of $\langle\mathrm{SNRP}\rangle$ with the increase of input power is evident for any strength of the diffuser and that of the turbulence. Therefore, for a particular communication link operating in weak turbulence there exists the optimal diffuser. For example, in Fig. 9 for $\mathrm{SNRC}_{0}=30 \mathrm{~dB}$ the best choice is the diffuser with $l_{c}$ $=1 \mathrm{~cm}$ (dotted curve), which corresponds to $\langle\mathrm{SNRP}\rangle$ $=25 \mathrm{~dB}$.

In the focusing regime, the power reduction caused by a partially coherent beam spreading starts to dominate the favorable effect of the flux variance (recall Fig. 7). Therefore, as shown in Fig. 10 stronger diffuser together with greater amount of transmitted power are required; here, for fixed $\mathrm{SNRC}_{0}=30 \mathrm{~dB}$ the diffuser with $l_{c}=1 \mathrm{~cm}$ (dotted curve) would not provide a sufficient level of BER; for $\mathrm{SNRC}_{0}=50 \mathrm{~dB}$ the diffuser with $l_{c}=1 \mathrm{~mm}$ (solid curve) should be used.

Based on Fig. 11, demonstrating $\langle\mathrm{SNRP}\rangle$ in strong turbulence we note that no matter how strong the diffuser, the required level 21 to $22 \mathrm{~dB}$ cannot be attained only with the use of partial coherence. Refer to Fig. 8 to see that in strong fluctuations the flux variance can not be sufficiently reduced. In this regime, only the combination of different tools (multiaperture receivers, adaptive optics, partial coherence) would be efficient for the mitigation of the atmospheric effects. 


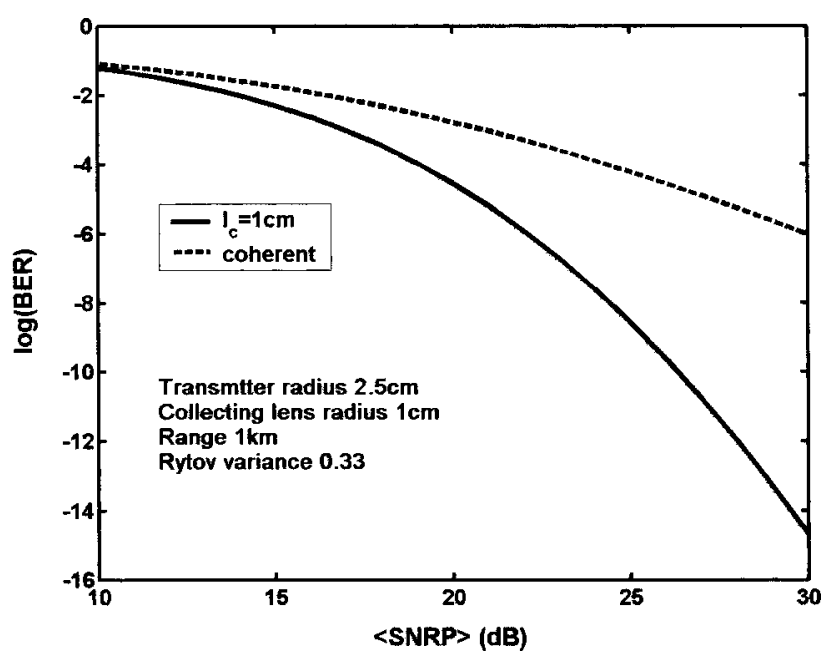

Fig. 12 BER of partially coherent beams (logarithmic scale) as a function of $\langle$ SNRP $\rangle$ (in decibels) in weak turbulence for coherent and partially coherent beams.

\subsection{BERs}

Following Ref. 34 the probability of error (BER) for a Gaussian beam (OOK modulation scheme) in the atmospheric turbulence can be based on the gamma-gamma probability density function of the intensity fluctuations for all irradiance fluctuation conditions, i.e.,

$$
\begin{aligned}
\operatorname{BER}= & \frac{(\alpha \beta)^{(\alpha+\beta) / 2}}{\Gamma(\alpha) \Gamma(\beta)} \int_{0}^{\infty} \operatorname{erfc}\left(\frac{\langle\mathrm{SNRP}\rangle x}{2 \sqrt{2}}\right) \cdot x^{(\alpha+\beta / 2)-1} \\
& \times K_{\alpha-\beta}\left[2(x a \beta)^{1 / 2}\right] \mathrm{d} x,
\end{aligned}
$$

where $\langle\mathrm{SNRP}\rangle$ is defined in Eq. (46); $K_{p}(x)$ is a $K$-Bessel function; $\operatorname{erfc}(x)$ is the complementary error function; and $\alpha$ and $\beta$ are two parameters of the distribution, related correspondingly to the large-scale $x$ and the small-scale $y$ intensity fluctuations:

$$
\alpha=\frac{1}{\exp \left(\sigma_{\ln x}^{2}\right)-1}, \quad \beta=\frac{1}{\exp \left(\sigma_{\ln y}^{2}\right)-1}
$$

where $\sigma_{\ln x}^{2}$ and $\sigma_{\ln y}^{2}$ are given by Eqs. (40) and (42).

Although the probability [Eq. (39)] is valid in all atmospheric conditions we concentrate primarily on weak and moderate regimes where the partial coherence is proving to be very efficient. Using the conventional way to display the system performance, we plot BER [Eq. (39)] versus $\langle\mathrm{SNRP}\rangle$ in decibel (see Fig. 12) for two beams: perfectly coherent (dashed curve) and partially coherent, with $l_{c}$ $=1 \mathrm{~cm}$ (solid curve) in weak turbulence $\left(\sigma_{1}^{2}=0.33\right)$. The later diffuser is actually on the order of the optimal diffuser for this particular scenario (refer to Fig. 9).

If the transmitted power of the operating system is fixed (can not be adjusted) the partially coherent beam can improve the BER up to several orders of magnitude over the distances under $1 \mathrm{~km}$ and values of $C_{n}^{2}$ of the order of $10^{-14} \mathrm{~m}^{-2 / 3}$, which corresponds to Rytov variance $\sigma_{1}^{2}<1$ (for example, in Fig. 12). This increase of BER is shown

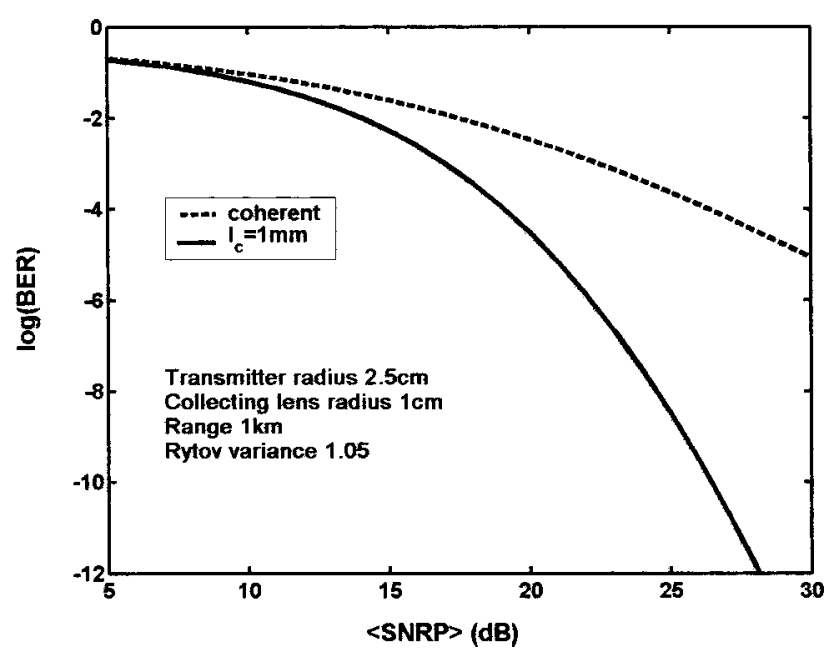

Fig. 13 BER of partially coherent beams (logarithmic scale) as a function of $\langle\mathrm{SNRP}\rangle$ (in decibels) in focusing regime for coherent and partially coherent beams.

here to be critical (BER attains the value $10^{-9}$ at $\langle\mathrm{SNRP}\rangle$ $=25 \mathrm{~dB}$, while a perfectly coherent beam can not produce this BER with any transmitted power). See Fig. 9 to find the corresponding values of the $\mathrm{SNRC}_{0}$ for each beam.

On the other hand if a certain system performance is needed (BER should be set up permanently to some level) then the diffuser can also be used as a tool for the reduction of the transmitting power.

In Fig. 13 the BER of a perfectly coherent beam (dashed curve) and a partially coherent beam with $l_{c}=1 \mathrm{~mm}$ (solid curve) are plotted in the focusing regime $\left(\sigma_{1}^{2}=1.05\right)$. This choice of the diffuser was discussed in Fig. 10. For $\langle\mathrm{SNRP}\rangle=25 \mathrm{~dB}$, the $\mathrm{BER}=10^{-9}$, but it requires very high level of input power $\left(\mathrm{SNRC}_{0}\right.$ should be at least $\left.50 \mathrm{~dB}\right)$.

In Fig. 14 the BER of the same two beams as in Fig. 13 are displayed versus $\langle\mathrm{SNRP}\rangle$ in strong turbulence $\left(\sigma_{1}^{2}\right.$ $=7.0)$. In this case, partial coherence still reduces the BER

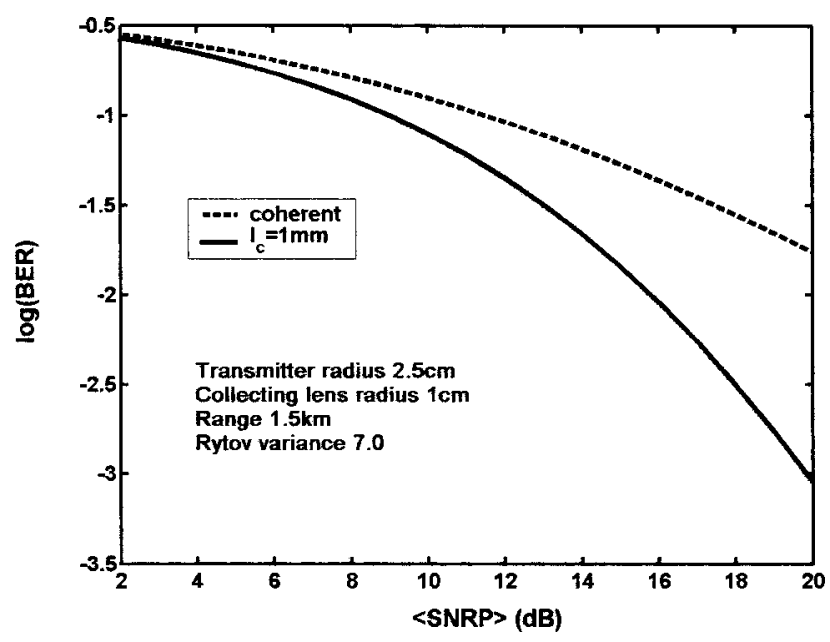

Fig. 14 BER of partially coherent beams (logarithmic scale) as a function of $\langle\mathrm{SNRP}\rangle$ (in decibels) in strong turbulence for coherent and partially coherent beams. 
but by an amount which is not sufficient for good system performance.

With the decrease of correlation distance $l_{c}$, parameter $\alpha$ given by Eq. (49) of the gamma-gamma distribution grows rapidly, which might cause some difficulties in numerical integration of Eq. (39). In such cases, a single Gamma distribution might be used instead; hence the BER [Eq. (39)] in such cases can be replaced by

$\mathrm{BER}=\frac{1}{2} \frac{\gamma^{\gamma}}{\Gamma(\gamma)} \int_{0}^{\infty} \operatorname{erfc}\left(\frac{\langle\mathrm{SNRP}\rangle x}{2 \sqrt{2}}\right) x^{\gamma} \exp (-\gamma x) \mathrm{d} x$,

where $\gamma$ is the parameter of the distribution, related to the flux variance [Eq. (39)] by

$$
\gamma=\frac{1}{\sigma_{\text {irrad }}^{2}\left(L+L_{f}, \Omega_{G}\right)} .
$$

The integral of Eq. (50) predicts slightly higher levels of the BER compared with that of Eq. (39). The solid curve in Fig. 13 was generated with the help of this simplification.

\section{Conclusions}

The model (based on the thin complex phase screen at the transmitter) for the analysis of the scintillation index of a partially coherent beam significantly differs from the conventional approach. However, our formulas proved to be consistent (sometimes even more accurate) with results known in the literature for all statistics of interest. ${ }^{35}$ For instance, the MCF of a partially coherent Gaussian beam (and other statistics based on it) was established in the weak atmospheric regime for the Kolmogorov spectrum model, improving results of Ref. 1. Moreover, our model is independent of the spectrum models for the phase screen and the atmosphere; therefore the effects of inner/outer scales can be also studied by changing the spectrum. ${ }^{32}$ On the other hand, the model would work for more complex optical systems; in Ref. 35, we illustrated this by deriving several statistics for the image plane of the receiver.

However, the MCF was derived mainly to establish the model. The real breakthrough of our approach is the possibility to model the scintillation index of a partially coherent beam for all atmospheric conditions. The connection between second order statistics and the scintillation index was made through the way of "effective" beam parameters, related to the degree of coherence. The longitudinal and radial components of the scintillation index were treated separately but both were studied as a function of Rytov variance and the degree of coherence. The corresponding plots (see Figs. 2-5) display the dependence of the scintillation index components on the degree of spatial coherence of the source including incoherent and coherent limits.

A developed simple algebraic expression for the longitudinal component of the scintillation index enabled us to analyze the performance of the lasercom channel (Sec. 5). In particular, the calculation of the SNR and BER was demonstrated in detail. For the analysis of the SNR (Sec. 5.1) one should take into account the relation [Eq. (46)] between the transmitted power and coherence length $l_{c}$ to optimize the later parameter where it is possible (in weak conditions, for short ranges). In moderate and strong atmospheric tur- bulence the strongest possible diffuser $\left(l_{c}\right.$ of the order of 1 $\mathrm{mm}$ ) would be the most effective. Based on the analysis of the BER we made a comparison between the results due to a coherent beam and the optimal partially coherent beam (based on the trade-off between the power loss and the flux variance reduction in Sec. 5.2) in weak, moderate, and strong atmospheric fluctuations. While in weak turbulence the BER is practically controllable by the value of $l_{c}$, in other situations, the improvement in BER up to several orders of magnitude was found with the use of a very strong diffuser (or quasi-incoherent source) with $l_{c}$ $=1 \mathrm{~mm}$.

\section{References}

1. J. C. Ricklin and F. M. Davidson, "Atmospheric turbulence effects on a partially coherent Gaussian Beam: implications for free-space laser communication," J. Opt. Soc. Am. A 19, 1794-1802 (2002).

2. G. Gbur and E. Wolf, "Spreading of partially coherent beams in random media," J. Opt. Soc. Am. A 19, 1592-1598 (2002).

3. V. G. Sidorovich, V. V. Ragulsky, M. V. Vasil'ev, A. A. Leshchev, and M. A. Sadovnikov, "Mitigation of aberration in a beam-shaping telescope and optical inhomogeneity in a free-space optical path using an extended light source coupled to the telescope," Proc. SPIE 4635, 179-191 (2002)

4. T. Shirai, A. Dogariu, and E. Wolf, "Directionality of some model beams propagating in atmospheric turbulence," Opt. Lett. 28, 610 (2003).

5. M. Salem, T. Shirai, A. Dogariu, and E. Wolf, "Long-distance propagation of partially coherent beams through atmospheric turbulence,' Opt. Commun. 216, 261 (2003).

6. T. Shirai, A. Dogariu, and E. Wolf, "Mode analysis of spreading of partially coherent beams propagating through atmospheric turbulence," J. Opt. Soc. Am. A 20, 1094 (2003).

7. A. Dogariu and S. Amarande, "Propagation of partially coherent beams: turbulence-induced degradation," Opt. Lett. 28(1), 10-12 (2003).

8. R. K. Tyson, "Bit-error rate for free-space adaptive optics laser communications," J. Opt. Soc. Am. A 19(4), 753-758 (2002).

9. J. C. Ricklin, and F. M. Davidson, "Atmospheric optical communication with a Gaussian Schell beam," J. Opt. Soc. Am. A 20, 856-866 (2003).

10. V. A. Banakh, V. M. Buldakov, and V. L. Mironov, "Intensity fluctuations of a partially coherent light beam in a turbulent atmosphere,' Opt. Spektrosk. 54, 1054-1059 (1983).

11. A. I. Kon and V. I. Tatarskii, "On the theory of the propagation of partially coherent light beams in a turbulent atmosphere," Radiophys. Quantum Electron. 15, 1187-1192 (1972).

12. J. W. Goodman, "Statistical properties of laser speckle patterns," in Laser Speckle and Related Phenomena, J. C. Dainty, Ed., SpringerVerlag, Berlin (1975).

13. J. Ohtsubo and T. Asakura, "Statistical properties of laser speckle produced in the diffraction field," Appl. Opt. 16, 1742-1753 (1977).

14. M. S. Belenkii, A. I. Kon, and V. L. Mironov, "Turbulent distortions of the spatial coherence of a laser beam,' Sov. J. Quantum Electron. 7, 287-290 (1977)

15. J. C. Leader, "Atmospheric propagation of partially coherent radiation," J. Opt. Soc. Am. 68, 175-185 (1978).

16. J. C. Leader, "Intensity fluctuations resulting from a spatially partially coherent light propagating through atmospheric turbulence," J. Opt. Soc. Am. 69, 73-84 (1979).

17. S. C. H. Wang and M. A. Plonus, "Optical beam propagation for a partially coherent source in the turbulent atmosphere," J. Opt. Soc. Am. 69, 1297-1304 (1979).

18. L. Fante, "Intensity fluctuations of an optical wave in a turbulent medium, effect of source coherence," Opt. Acta 28, 1203-1207 (1981).

19. T. Friberg and R. L. Sudol, "Propagation parameters of Gaussian Schell-model beams," Opt. Commun. 41, 383-387 (1982).

20. Y. Baykal, M. A. Plonus, and S. J. Wang, "The scintillations for weak atmospheric turbulence using a partially coherent source," Radio Sci. 18, 551-556 (1983)

21. Y. Baykal and M. A. Plonus, "Intensity fluctuations due to a spatially partially coherent source in atmospheric turbulence as predicted by Rytov's method," J. Opt. Soc. Am. A 2, 2124-2132 (1985).

22. T. Yoshimura, "Statistical properties of dynamic speckles," J. Opt. Soc. Am. A 3, 1032-1054 (1986).

23. H. T. Yura, S. G. Hanson, and T. P. Grum, "Speckle statistics and interferometric decorrelation effects in complex $A B C D$ optical systems," J. Opt. Soc. Am. A 10, 316-323 (1993).

24. H. T. Yura, S. G. Hanson, and L. Lading, "Laser Doppler velocimetry: 
analytical solution to the optical system including the effects of partial coherence of the target," J. Opt. Soc. Am. A 12, 2040-2047 (1995).

25. L. Mandel and E. Wolf, Optical Coherence and Quantum Optics, Cambridge University Press, Cambridge (1995)

26. A. C. Schell, "The multiple plate antenna," PhD Dissertation, Massachusetts Institute of Technology, Cambridge (1961)

27. S. Wandzura, "Meaning of quadratic structure functions," J. Opt. Soc. Am. 70, 745-747 (1980)

28. R. L. Fante, "Some physical insights into beam propagation in strong turbulence," Radio Sci. 15, 757-762 (1980).

29. V. A. Banakh and V. M. Buldakov, "Effect of the initial degree of spatial coherence of a light beam on intensity fluctuations in a turbulent atmosphere," Opt. Spektrosk. 55, 707-712 (1983).

30. V. A. Banakh, V. M. Buldakov, and V. L. Mironov, "Strong fluctuations of the incoherent radiation flux in a turbulent atmosphere," $R a-$ diofiz. 24(6), 703-708 (1981) (in Russian).

31. J. W. Goodman, Statistical Optics, Wiley, New York (1985).

32. L. C. Andrews and R. L. Phillips, Laser Beam Propagation through Random Media, SPIE Press, Bellingham, WA (1998).

33. L. C. Andrews, R. L. Phillips, and A. R. Weeks, "Propagation of a Gaussian-beam wave through a random phase screen," Waves Random Media 7, 229-244 (1997).

34. L. C. Andrews, R. L. Phillips, and C. Y. Hopen, Laser Beam Scintillation with Applications, SPIE Press, Bellingham, WA (2001).

35. O. Korotkova, L. C. Andrews, and R. L. Phillips, "Speckle propagation through atmospheric turbulence: effects of a random phase screen at the source," Proc. SPIE 4821, 98-109 (2002).

36. L. C. Andrews, Special Functions of Mathematics for Engineers, SPIE Press, Bellingham, WA (1998).

37. L. C. Andrews, M. A. Al-Habash, C. Y. Hopen, and R. L. Phillips, "Theory of optical scintillation: Gaussian-beam wave model," Waves Random Media 11, 271-291 (2001).

38. O. Korotkova, L. C. Andrews, and R. L. Phillips, "Phase diffuser at the transmitter for lasercom link: effect of partially coherent beam on the bit-error rate," Proc. SPIE 4976, 70-77 (2003).

39. J. H. Shapiro, "Imaging and optical communication through atmospheric turbulence," in Laser Beam Propagation in the Atmosphere, J. Strohbehn, Ed., Springer, New York (1978).

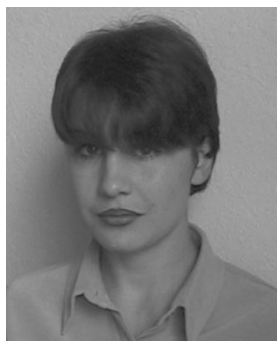

Olga Korotkova is currently with the University of Central Florida, Department of Mathematics. She received her BA degree in applied mathematics in 1999 from Samara State University, Russia, and her MS degree in 2002 and her PhD in 2003 in mathematical sciences from the University of Central Florida. Her research interests are propagation and scattering of optical waves in random media (in particular, in atmospheric turbulence), laser radars, and free-space optical communication systems.

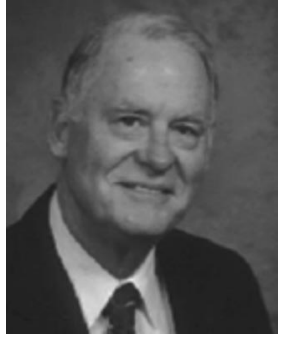

Larry C. Andrews is a professor of mathematics with the University of Central Florida and an associate member of the School of Optics Center for Research and Education Optics and Lasers (CREOL). He is also an associate member of the Florida Space Institute (FSI). Previously he held a faculty position at Tri-State University and was a staff mathematician with the Magnavox Company, antisubmarine warfare (ASW) operation. He received a $\mathrm{PhD}$ degree in theoretical mechanics in 1970 from Michigan State University. Dr. Andrews has been an active researcher in optical wave propagation through random media for more than 20 years and is the author or coauthor of several textbooks on differential equations, boundary value problems, special functions, integral transforms, and wave propagation through random media. Along with wave propagation through random media, his research interests include special functions, random variables, atmospheric turbulence, and signal processing.

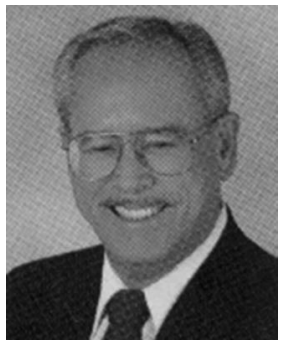

Ronald L. Phillips directs the Florida Space Institute (FSI) and is a professor in the Department of Electrical and Computer Engineering, the University of Central Florida. Dr. Phillips is also a member of the Department of Mathematics and an associate member of the Center for Research and Education in Optics and Lasers (CREOL). He has held positions on the faculties at Arizona State University and the University of California, San Diego. He received a PhD degree in electrical engineering in 1970 from Arizona State University. Dr. Phillips has been an active researcher in wave propagation through random media for more than 22 years. He was awarded a senior North Atlantic Treaty Organization (NATO) postdoctoral fellowship in 1977 and the American Society for Engineering Education 1983 Medal for outstanding contributions in research. Dr. Phillips is coauthor of two textbooks on wave propagation through random media and, in addition to optical wave propagation, his research interests include optical communications and imaging through atmospheric turbulence. 